\title{
Período reprodutivo de fêmeas de Hypostomus commersoni Valenciennes, 1836, em uma barragem na bacia do rio Uruguai
}

\author{
Rita Cristina Gomes Galarça \\ Iara Terezinha Garcia Müller \\ Herbert Spencer Filho \\ Romualdo Elias Soares \\ Paula Cunha \\ Thiago Signori Gralha \\ Edward Frederico Castro Pessano * \\ Marcus Vinícius Morini Querol \\ Universidade Federal do Pampa \\ Núcleo de Pesquisas Ictiológicas, Limnológicas e Aquicultura da Bacia do Rio Uruguai \\ BR 472, km 593, CEP 97500-970, Uruguaiana - RS, Brasil \\ * Autor para correspondência \\ edwardpessano@unipampa.edu.br
}

Submetido em 25/07/2013

Aceito para publicação em 18/03/2014

\section{Resumo}

O cascudo Hypostomus commersoni, nativo da Bacia do Rio Uruguai, pertencente à família Loricariidae, é encontrado habitualmente em ambientes lênticos e tem sua biologia ainda pouco conhecida na região do Pampa Brasileiro. Foi verificado o período reprodutivo pela análise da variação mensal do índice gonadossomático (IGS), do índice hepatossomático (IHS), e o fator de condição gonadal $(\Delta \mathrm{K})$, além da observação macroscópica do estádio de desenvolvimento gonadal. Após a análise dos resultados pode se concluir que a espécie em estudo tem seu período reprodutivo de setembro estendendo-se a janeiro, atingindo seu pico de reprodução em novembro. Os indicadores IHS e $\Delta \mathrm{K}$ apresentaram-se mais elevados no mês de outubro, demonstrando uma melhor condição gonadal e o IHS revelou um acúmulo de reservas lipídicas para fornecer energia na vitelogênese, no mês antecedente ao pico de reprodução, sendo desta forma, bons indicadores que corroboram com o período de reprodução determinado.

Palavras-chave: Loricarridae; Maturação gonadal; Pampa Brasileiro

\section{Abstract}

Reproductive period of female Hypostomus commersoni Valenciennes, 1836, in a dam reservoir in the Pampa Brazilian. Hypostomus commersoni belongs to the family Loricariidae and is native to the Uruguay River Basin. It is commonly found in lentic environments, and their biology is poorly known in the Pampa regions of Brazil. The reproductive period was determined by analysis of the gonadosomatic index (GSI), hepatosomatic 
index (HSI), and condition factor $(\Delta \mathrm{K})$, in addition to the macroscopic observation of the gonadal development stage. The results indicated that the species under study had a reproductive period lasting from September to January, with reproduction peaking in November. $\Delta \mathrm{K}$ and HSI were highest in October, showing respectively a better gonadal condition and an accumulation of lipid reserves to provide energy for vitellogenesis, in the month preceding peak breeding. Thus, $\Delta \mathrm{K}$ and HSI are good indicators of the reproduction period of H. commersoni.

Key words: Loricarridae; Gonadal Maturation, Brazilian Pampa

\section{Introdução}

Hypostomus commersoni é uma espécie de peixe conhecida popularmente como cascudo, que habita costumeiramente ambientes lênticos dulcícolas nas bacias do Paraná, Uruguai e Laguna dos Patos da América do Sul, podendo atingir até de 42,5 cm de comprimento (REIS, et al., 2003). O gênero Hypostomus possui cerca de 110 espécies colonizando todos os habitats aquáticos da América do Sul (MONTOYA-BURGOS, 2003). Hypostomus commersoni pertence à família Loricariidae e é caracterizada por apresentar corpo quase completamente coberto por placas ósseas e boca ventral em forma de ventosa (CASTELLANOS, 1991). Compreende a ordem Siluriforme que corresponde a segunda maior ordem entre os Teleósteos quanto ao número de espécies, aproximadamente 70 gêneros e 690 espécies (REIS et al., 2003).

Embora existam trabalhos sobre o ciclo reprodutivo da espécie, pouco se conhece seu comportamento na região de Uruguaiana, Fronteira Oeste do RS, na bacia do rio Uruguai Médio. Desta forma a investigação poderá contribuir para a sua preservação e no gerenciamento pesqueiro local.

Barbieri (1994) destaca que estudos reprodutivos ocupam importante lugar na investigação pesqueira, favorecendo subsídios à elaboração de programas visando à exploração racional e à preservação das espécies ícticas de rios e lagos.

Dias et al. (1998) destacam que a utilização de indicadores quantitativos da maturação gonadal, tais como o índice gonadossomático, tem servido de parâmetros para a definição de uma escala de maturidade, que é fundamental para compreender o ciclo reprodutivo e prever o comportamento de uma população durante o ano. Sabe-se que o ciclo reprodutivo dos peixes passa por diferentes fases de desenvolvimento gonadal, pois a gônada, ao amadurecer, sofre profundas modificações e pode caracterizar fases durante o seu desenvolvimento. Conforme Vazzoler (1996), nas fases finais, verifica-se um marcado aumento no volume e, consequentemente, no peso dos ovários e adicionalmente, o IGS é um indicador quantitativo utilizado para avaliar o período de reprodução da espécie.

Portanto, esta investigação objetivou estudar o período reprodutivo das fêmeas de $H$. commersoni, através da observação dos estádios de maturação gonadal, da análise de variação mensal do índice gonadossomático (IGS), do índice hepatossomático (IHS) e o fator de condição gonadal $(\Delta \mathrm{K})$ durante um ciclo anual. Desta forma, o estudo contribui fornecendo informações que podem ser utilizadas futuramente para o manejo da espécie como, por exemplo, manejo pesqueiro.

\section{Material e Métodos}

A pesquisa foi desenvolvida no Núcleo de Pesquisas Ictiológicas, Limnológicas e Aquicultura da Bacia do Rio Uruguai (NUPILABRU) da Universidade Federal do Pampa (UNIPAMPA), Campus Uruguaiana, Rio Grande do Sul, Brasil, investigando o período reprodutivo de fêmeas de $H$. commersoni.

As coletas dos animais foram realizadas mensalmente na barragem da Universidade Federal do Pampa (Figura 1), localizada a $29^{\circ} 50^{\prime} 0.1$ "S de latitude e $57^{\circ} 05$ '53.86"O de longitude, a qual possui aproximadamente 10 ha de área alagada. Essa barragem é abastecida e desemboca no arroio Felizardo, percorrendo uma distância de $6,5 \mathrm{~km}$, até a conjunção com o arroio Itapitocai, que deságua no rio Uruguai. 
FIGURA 1: Mapa da localidade de estudo onde os exemplares foram coletados.

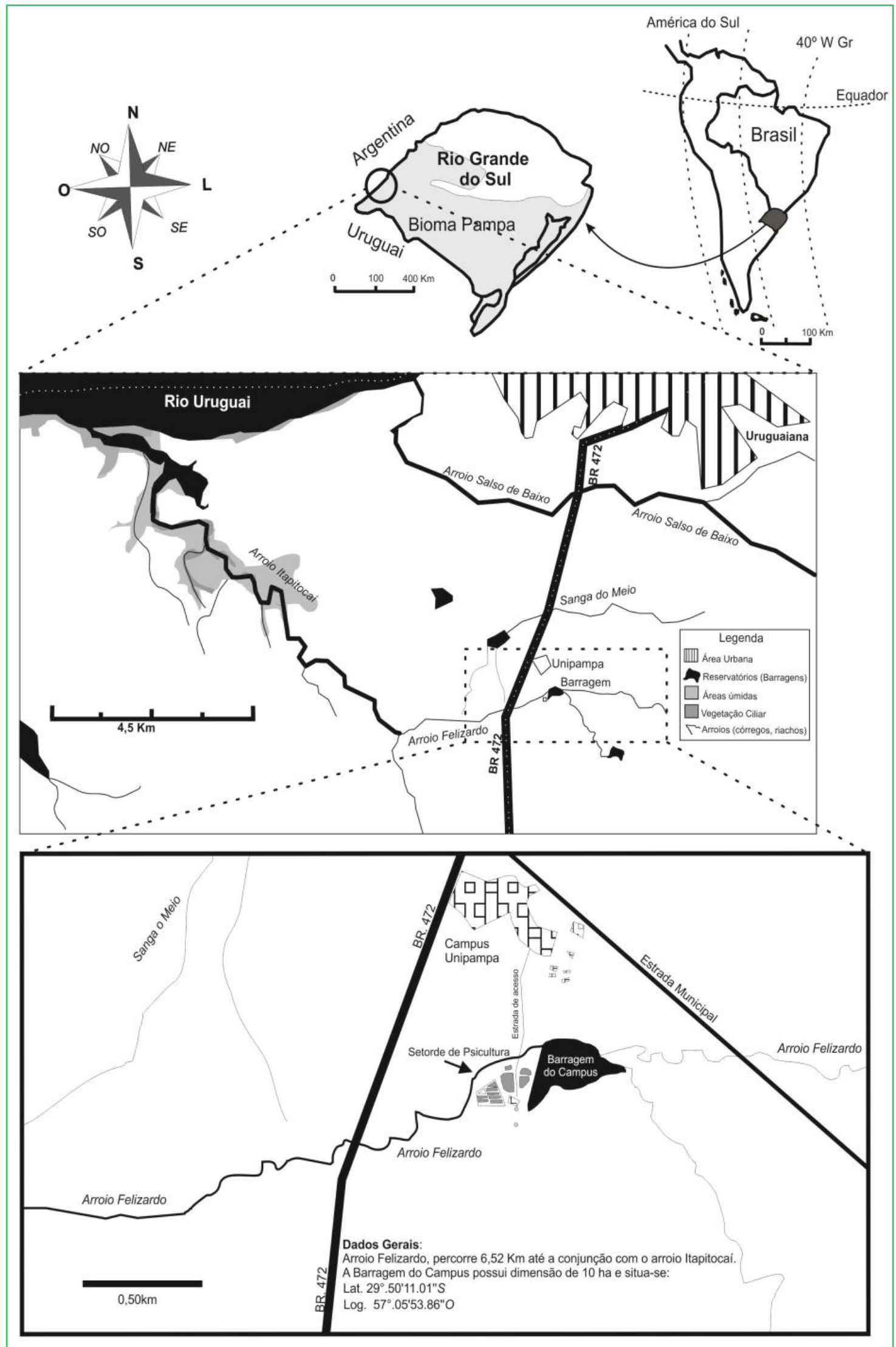


As investigações ocorreram durante o período de fevereiro de 2010 a janeiro de 2011. Após as coletas, os indivíduos capturados foram analisados no laboratório de Biologia e Ecologia de peixes do NUPILABRU. Para as coletas, foram utilizados os seguintes petrechos de pesca: uma rede de malhas de $1,5 \mathrm{~cm}$; uma rede de malha de 2,0 cm; uma rede de 3,0 cm; uma rede de 4 $\mathrm{cm}$ e uma rede $5 \mathrm{~cm}$, de entre-nós adjacentes. Todas as redes possuíam um comprimento de $2 \mathrm{~m}$ de altura e 50 $\mathrm{m}$ de comprimento. Também foram utilizadas peneiras e uma rede do tipo picaré (arrasto). Em cada ocasião de coleta foram passadas as peneiras nas margens da barragem junto a vegetação aquática, com o objetivo de capturar indivíduos em recrutamento, larvas e alevinos da espécie. As redes foram armadas em diferentes locais da barragem por um período de $24 \mathrm{~h}$, em vários pontos da mesma. Os exemplares foram fixados em formol a $10 \%$, e, no laboratório, medidos, pesados e eviscerados, tendo as gônadas conservadas em álcool 70\%, e o fígado descartado.

A verificação dos parâmetros físico-químicos da água da barragem (temperatura, oxigênio dissolvido, amônia, nitrito, condutividade e salinidade) foi realizada mensalmente, juntamente com a realização das coletas, através da utilização de um aparelho multiparâmetro portátil marca Hanna, modelo HI 9828. As análises tiveram o objetivo de monitorar os referidos parâmetros, bem como de acompanhar a variação da qualidade da água a partir dos fatores abióticos, promovendo uma observação dos mesmos para com o período reprodutivo do animal.

O material coletado após as análises foi depositado na coleção científica do NUPILABRU.

Para cada exemplar, registrou-se o sexo, o comprimento padrão (Ls), da ponta do focinho até o pedúnculo caudal, com precisão de $0,1 \mathrm{~cm}$, o peso total $(\mathrm{Wt})$, peso das gônadas $(\mathrm{Pg})$ e peso do fígado $(\mathrm{Pf}) \mathrm{com}$ precisão de $0,01 \mathrm{~g}$.

O sexo e os estádios de desenvolvimento gonadal foram determinados pela observação macroscópica das gônadas, conforme Vazzoler (1996).

O período reprodutivo foi estabelecido por meio da análise do fator de condição e do índice hepatossomático e da comparação destes parâmetros com a curva de maturação, elaborada com base na variação mensal do índice gonadossomático médio.

Os cálculos do índice gonadossomático (IGS), do índice hepatossomático (IHS) e do fator de condição sob a influência do peso das gônadas $(K)$ e do fator de condição sem o peso das gônadas (K') e do fator de condição gonadal $(\Delta \mathrm{K})$ foram efetuados conforme Vazzoler $(1981 ; 1996)$ :

$$
\mathrm{IGS}=(\mathrm{Wg} / \mathrm{Wt}) .100 ; \mathrm{K}=\mathrm{Pt} / \mathrm{Cpb} \text {; }
$$

$\mathrm{K}^{\prime}=\mathrm{Pc} / \mathrm{Cpb} ; \mathrm{Pc}=\mathrm{Pt}-\mathrm{Pg} ; \mathrm{Pt}$, peso total $(\mathrm{g}) ; \mathrm{Pc}$, peso total menos o peso da gônada;

b, coeficiente de alometria obtido através da relação peso-comprimento;

$$
\Delta \mathrm{K}=\mathrm{K}-\mathrm{K}^{\prime} ; \mathrm{IGS}=(\mathrm{Pg} / \mathrm{Pt}) .100
$$

O coeficiente angular da relação peso-comprimento (b) foi estimado através da equação de regressão entre as variáveis de peso total e comprimento padrão, determinado pelo modelo de regressão $y=\exp \left(a+b^{*} x\right)$ no software Statgraf.

\section{Resultados}

Foram capturados 89 indivíduos, fêmeas, durante o ciclo anual, através de amostragens mensais. O coeficiente angular b $(3,65647)$, que expressa a relação peso-comprimento e foi obtido pela equação $\mathrm{Wt}=\exp$ $(0,0939313+3,65647 \times \mathrm{Ls})$, foi determinante para a obtenção dos índices de $\mathrm{K}, \mathrm{K}$ ' e $\Delta \mathrm{K}$, conforme observado na Figura 2.

Através da observação gonadal, foi encontrado para as fêmeas de $H$. commersoni maior número de indivíduos no estádio em maturação e maduro entre setembro e janeiro (primavera/verão), conforme pode ser visualizado na Tabela 1 . Observa-se alta frequência de indivíduos em maturação inicial no período de março a agosto (outono/inverno), porém, o período reprodutivo teve seu início na primavera. A frequência de indivíduos atrésicos (dezembro a fevereiro) aparece acompanhando os indivíduos maduros e também, posteriormente. Foram encontradas fêmeas imaturas nas diferentes épocas do ano. Outubro e novembro foi o período onde foi encontrada maior frequência de fêmeas maduras, aptas à desova. 
FIGURA 2: Correlação do peso total e comprimento padrão de fêmeas de $H$. commersoni, coletados na barragem da Unipampa, Uruguaiana, RS, Brasil.

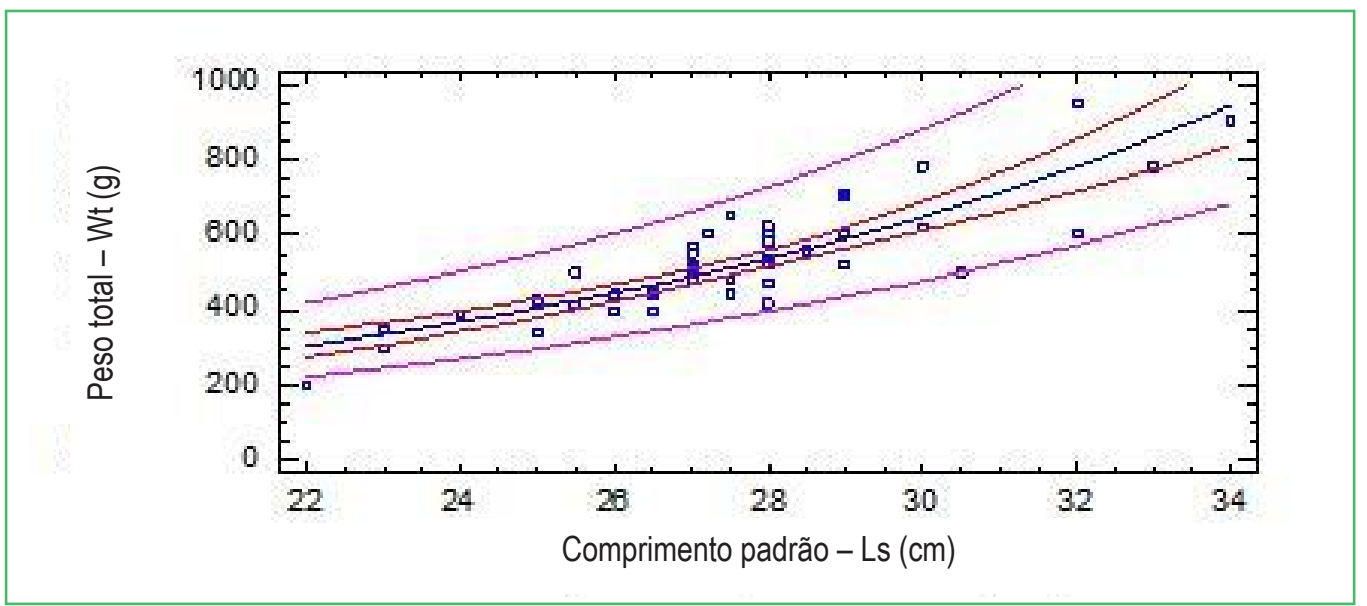

TABELA 1: Frequência dos estádios de maturação gonadal de H. commersoni durante o período de fevereiro de 2010 a janeiro de 2011, coletados na barragem da Unipampa, Uruguaiana, RS, Brasil.

\begin{tabular}{ccccccc}
\hline \multirow{2}{*}{ Período } & \multicolumn{7}{c}{ Fase de Desenvolvimento Gonadal } \\
\cline { 2 - 6 } & \multirow{2}{*}{ Imaturo } & Inicial & Intermediária & Final & \multirow{2}{*}{ Maduro } & \multirow{2}{*}{ Atrésico } \\
\cline { 2 - 6 } Fevereiro & $33 \%$ & - & - & - & - & - \\
Março & $67 \%$ & $33 \%$ & - & - & - & - \\
Abril & $40 \%$ & $60 \%$ & - & - & - & - \\
Maio & $25 \%$ & $75 \%$ & - & - & - & - \\
Junho & $25 \%$ & $75 \%$ & - & - & - & - \\
Julho & $22 \%$ & $78 \%$ & - & - & - & - \\
Agosto & $20 \%$ & $35 \%$ & $45 \%$ & - & $6 \%$ & - \\
Setembro & $10 \%$ & $20 \%$ & $30 \%$ & $34 \%$ & $67 \%$ & - \\
Outubro & - & - & - & $33 \%$ & $89 \%$ & - \\
Novembro & - & - & - & $11 \%$ & $40 \%$ & $20 \%$ \\
Dezembro & - & - & - & $40 \%$ & $16 \%$ & $35 \%$ \\
Janeiro & $40 \%$ & - & - & $9 \%$ & - \\
\hline
\end{tabular}

Fonte: Dados da Pesquisa

Pela análise mensal dos resultados do IGS das fêmeas (Figura 3), houve pequena elevação a partir de julho, notando-se índices superiores entre os meses de setembro e janeiro, atingindo seu pico de reprodução em novembro.
O Índice Hepatossomático (Figura 4) apresentou duas variações durante o ciclo anual, uma menor, no período que antecede o inverno (abril) e outra maior, no período imediatamente anterior o pico de desova (outubro). 
FIGURA 3: Índice Gonadossomático de fêmeas de H. commersoni no período de fevereiro de 2010 a janeiro de 2011 , coletados na barragem da Unipampa, Uruguaiana, RS, Brasil (média \pm desvio padrão).

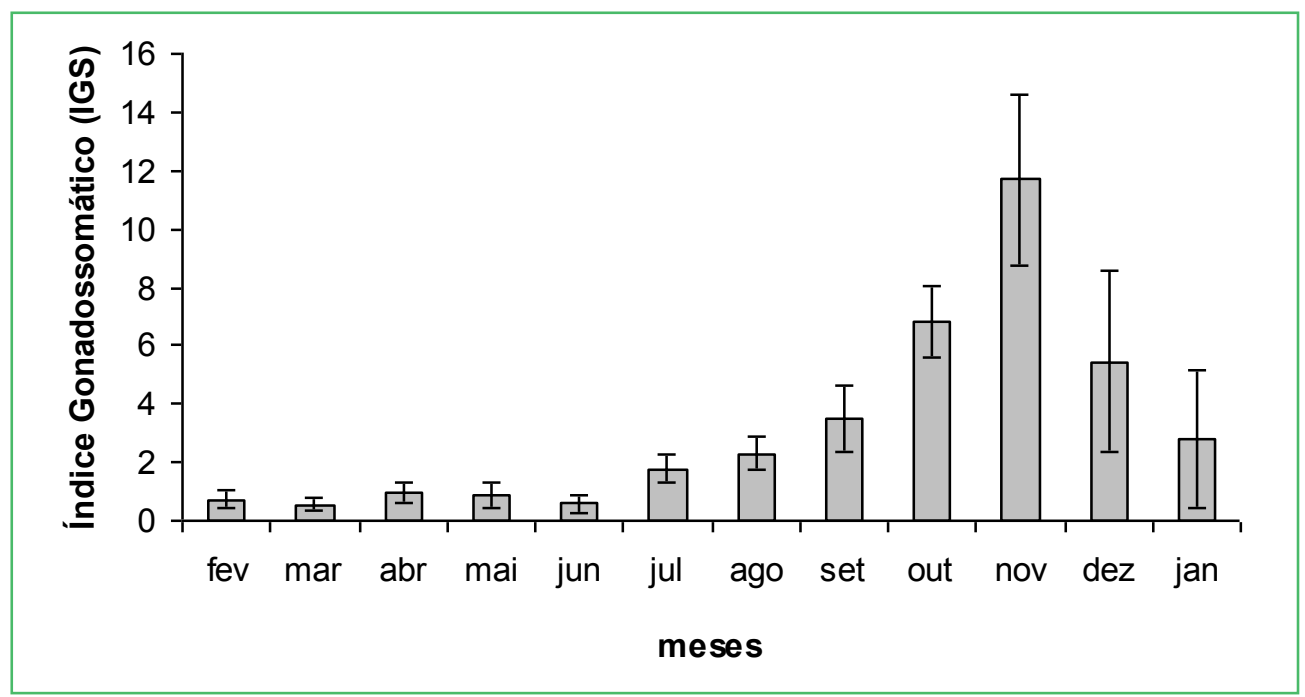

FIGURA 4: Índice Hepatossomático de fêmeas de H. commersoni no período de fevereiro de 2010 a janeiro de 2011 , coletados na barragem da Unipampa, Uruguaiana, RS, Brasil (média \pm desvio padrão).

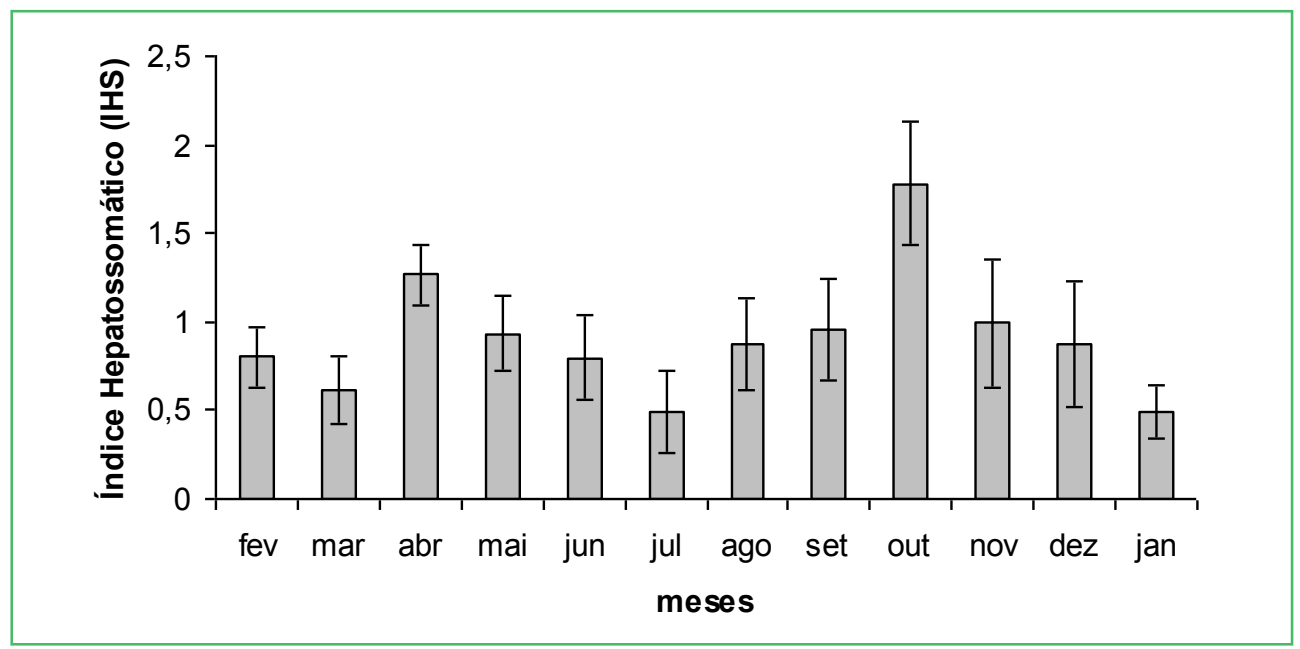

O fator de condição gonadal (Figura 5) apresentou elevação a partir de julho, porém, com maior elevação de setembro a janeiro, atingindo seu pico máximo em novembro.

A temperatura (Figura 6) apresentou elevação a partir do mês de agosto, quando atinge $20^{\circ} \mathrm{C}$.

Os parâmetros físico-químicos da água da barragem da UNIPAMPA durante o período de coleta encontraram-se dentro da normalidade, não havendo alteração que possa ter implicado ou determinado o período reprodutivo. Os valores obtidos foram: Oxigênio dissolvido: de 5,33 a 9 mgL; $\mathrm{pH}$ de 6, 67 a 7,6; amônia se manteve constante em $0,025 \mathrm{mgL}$; nitrito de 0 a $0,017 \mathrm{mgL}$; condutividade $0,076 \mathrm{mS} / \mathrm{cm}$ e salinidade $0,04 \mathrm{mgL}$. 
FIGURA 5: Fator de condição gonadal $(\Delta \mathrm{K})$ de fêmeas de $H$. commersoni no período de fevereiro de 2010 a janeiro de 2011 , coletados na barragem da Unipampa, Uruguaiana, RS, Brasil (média \pm desvio padrão).

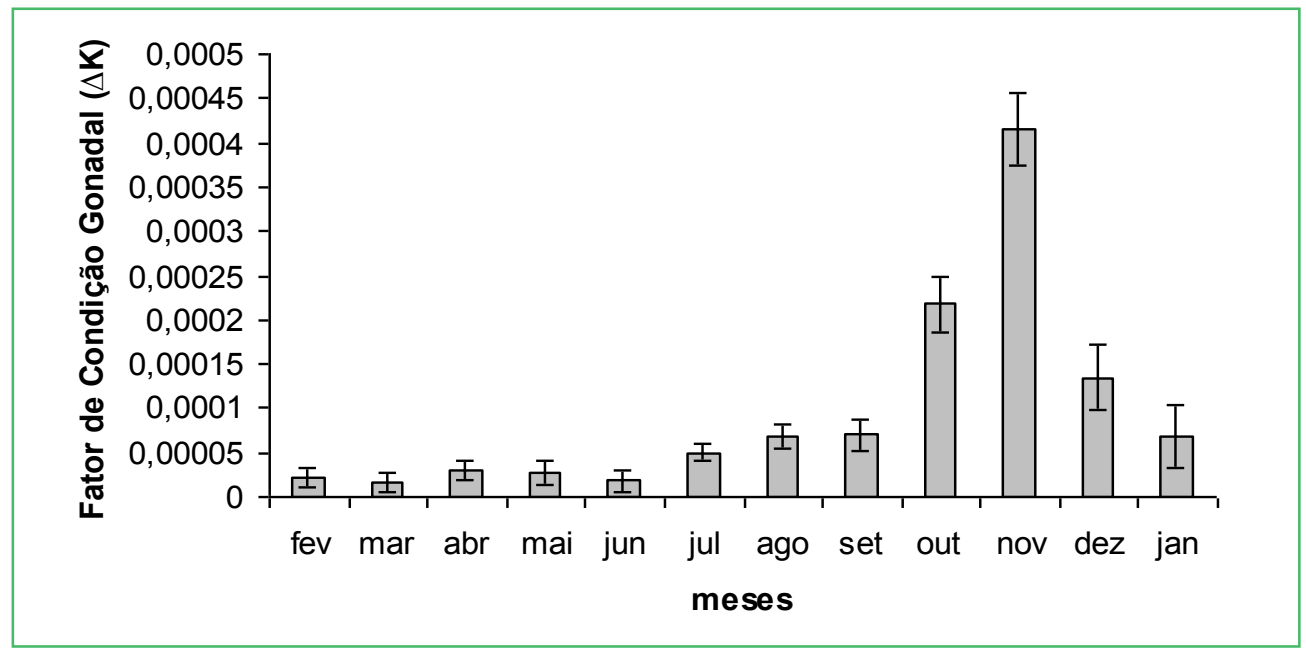

FIGURA 6: Temperatura da água e Temperatura atmosférica média, mínima e máxima em ${ }^{\circ} \mathrm{C}$ entre o período de fevereiro de 2010 a janeiro de 2011, coletados na barragem da Unipampa, Uruguaiana, RS, Brasil.

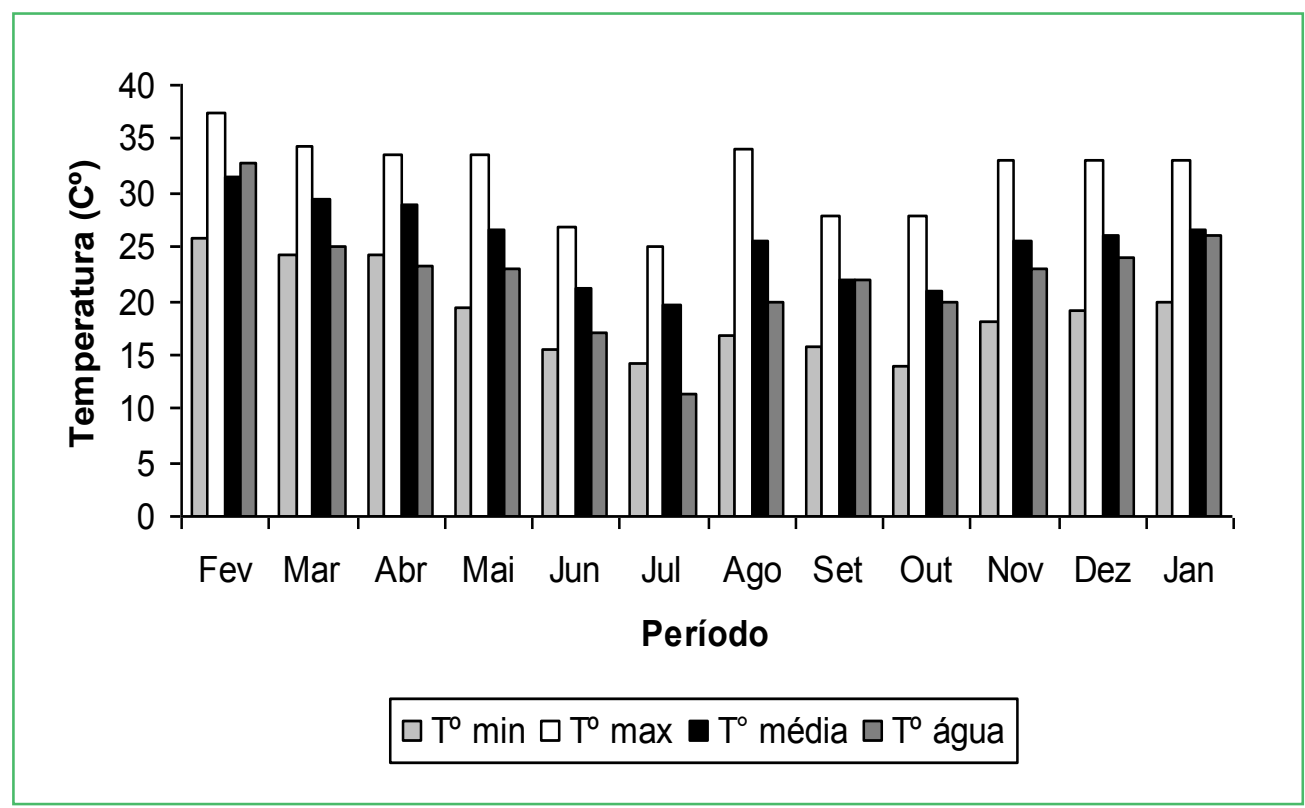

Fontes: Estação Meteorológica da PUC Uruguaiana; Boletim do Estado do RS - DISME/INMET; CPPMet/ UFPEL.

\section{Discussão}

O número de indivíduos coletados e analisados para o local de estudo, representam de forma significativa a população de $H$. commersoni, no referido ambiente investigado, pois, de acordo com Azevedo et al. (2003) e Pessano e. al. (2004), estudando o arroio Felizardo, córrego que alimenta hidricamente a barragem avaliada, a área de estudo possui uma densidade populacional estimada de 39 indivíduos por hectare, tendo maior ocorrência durante as estações de primavera e verão, assemelhando-se com os dados encontrados neste trabalho. Assim, de acordo com o número de indivíduos de H. commersoni coletados e utilizados neste estudo, é possível determinar o período de reprodutivo para as fêmeas nesta localidade. 
Em relação ao processo reprodutivo, destaca-se que as gônadas dos peixes de ciclo reprodutivo anual são, de um modo geral, submetidas à variações marcantes de volume e peso devido ao avanço do processo de maturação ovocitária (COSTA, et al, 2005). Essas variações determinam os estádios de maturação gonadal, que para $H$. commersoni vieram de encontro aos índices encontrados de IGS, onde pode se observar a partir de setembro, a fase desenvolvimento final das gônadas encontrando os indivíduos em período reprodutivo até o mês de janeiro. Ainda registra-se para o mês de novembro o período em que se encontra o maior número de indivíduos maduros.

A primeira elevação do IHS pode ser explicada pelo fato desse índice estar relacionado ao acúmulo de reservas energéticas para o período de inverno (QUEROL et al., 2002; 2004), portanto, o animal tende a acumular gordura para superar esse período de baixa atividade metabólica devido às temperaturas baixas ocorrentes na região. Já a elevação do índice do IHS em período anterior à desova, ocorre devido ao fígado estar relacionado com a mobilização de reservas energéticas necessárias à vitelogênese, o que também é verificado por Bazzoli et al. (1998) e Agostinho et al. (1990). Querol et al. (2002) destacam que o IHS pode ser utilizado como indicador do período reprodutivo quando associado a índices como o IGS, tornando-se, portanto, um bom indicador.

O Fator de Condição fornece importantes informações sobre o estado fisiológico dos animais, a partir do pressuposto de que indivíduos com relativamente maior massa corporal estão em melhor condição física (LIMA-JUNIOR e GOITEIN, 2006). O fator de condição analisado em função do estádio de maturação gonadal no período, corrobora a identificação dos estádios de desenvolvimento ovariano, conforme Vaz dos Santos et al. (2009). Os maiores valores de $\Delta \mathrm{K}$ e de IGS e a maior frequência de gônadas maduras ocorreram em novembro, indicando o pico reprodutivo das fêmeas. O IHS se mostrou mais elevado em outubro, mês que antecede o pico de elevação do IGS. Essa elevação ocorre, devido a reprodução ser considerada um processo altamente custoso energeticamente, e ocorre somente quando os animais estão situados em sua zona de conforto (RIBEIRO e MOREIRA, 2012).
Quando analisamos os parâmetros limnológicos encontrados e sua relação com o período reprodutivo, destaca-se que não foram encontradas correlações significativas, embora o aumento da temperatura ambiental coincidiu com o período de reprodução das fêmeas, fator que já foi verificado na literatura por Querol et al.(2004), quando avaliou a influência dos fatores abióticos sobre a reprodução do cascudo Loricariichtys platymetopon.

Assim, com a elevação da temperatura atmosférica e da água a partir do mês de agosto, favoreceu o processo de maturação gonadal, onde no mês de novembro, o IGS apresentou seu pico máximo, quando a temperatura esteve acima de $20^{\circ} \mathrm{C}$. De acordo com Colares de Mello (1989), a temperatura é um dos principais fatores abióticos que exercem influência na maturação gonadal. A influência dessa elevação térmica nos peixes também foi observada por Marcucci et al. (2005), ao estudar Loricariichthys platymetopon na região média do rio Paranapanema, ao verificarem que a reprodução ocorreu principalmente nos meses quentes. Agostinho et al. (1991) afirmam que a maturação gonadal do $H$. commersoni ocorre quando a temperatura média é superior a $18^{\circ} \mathrm{C}$. Vazzoler (1996) destaca que a maturação sexual, que inicia num período anterior ao das desovas, só é concluída quando o ambiente apresentar as condições ideais para a fecundação e desenvolvimento da prole.

O período reprodutivo apresentado por $H$. commersoni no presente estudo se assemelha ao verificado por Agostinho et al. (1991) e por Mega e Bemvenuti (2006), para esta mesma espécie, na região do Paraná e na Lagoa Mangueira, Santa Vitória do Palmar (RS), respectivamente, que encontraram o período reprodutivo ocorrendo entre novembro e fevereiro. Outros trabalhos revelam resultados semelhantes aos obtidos pelo $H$. commersoni para outras espécies da família Loricariidae (QUEROL et al., 2002) encontraram em Loricariichthys platymetopon, em Uruguaiana/RS, período reprodutivo entre novembro e fevereiro; Ramos e Konrad (1999) encontraram período de desova para Hypostomus strigaticeps entre os meses de setembro e fevereiro na região do rio Corumbataí em São Paulo; Viana et al. (2008) obtiveram para Hypostomus cf. ancistroides, no Paraná, período reprodutivo entre os 
meses de outubro a janeiro com desenvolvimento gonadal a partir de agosto, com pico em outubro. Hirschmann et al. (2011) verificaram que Hemiancistrus punctulatus apresentou período reprodutivo entre outubro e fevereiro. Nota-se, portanto, que o $H$. comersoni possui comportamento reprodutivo muito parecido às demais espécies comparadas, correspondendo à primavera e ao verão no Hemisfério Sul.

Desta forma, os dados encontrados no presente estudo colaboram com a perspectiva para a biologia da reprodução do $H$. commersoni na região. $\mathrm{O}$ que pode contribuir na preservação dos indivíduos em seu ecossistema natural e favorecem o conhecimento deste aspecto ecológico para a localidade estudada, bem como o desenvolvimento de outros trabalhos relacionados a um possível cultivo da espécie.

\section{Agradecimentos}

Ao Núcleo de Pesquisas Ictiológicas, Limnológica e Aquicultura da Bacia do Rio Uruguai - NUPILABRU, a FINEP, FAPERGS, aos professores, técnicos e alunos do Curso de Aquicultura (UNIPAMPA), que estiveram presentes atendendo solicitações e apoiando a pesquisa.

\section{Referências}

AGOSTINHO A. A.; BARBIERI, G.; VERANI, J. R.; HAHN, Y. N. S. Variação do fator de condição e do índice hepatossomático e sua relação com o ciclo reprodutivo em Rhinelepis aspera (Agassi, 1829) (Ostechthyes, Loricariidae) no rio Paranapanema. Ciência e Cultura, São Paulo, v. 42, n. 9, p. 711-714, 1990.

AgOstinho, A. A.; HAHN, N. S.; AGOSTINHO, C. S. Ciclo reprodutivo e primeira maturação de fêmeas de Hypostomus commersonii (Valenciennes, 1840) (Siluriformes, Loricariidae) no reservatório Capivari-Cachoeira, PR. Revista Brasileira de Biologia, São Carlos, v. 51, n. 1, p. 31-37, 1991.

AZEVEDO, C.; PESSANO, E.; TOMASSONI, D.; QUEROL, M. V.; QUEROL E. Diversidade específica, densidade e biomassa da ictiofauna da nascente do arroio Felizardo, Bacia do rio Uruguai médio, Uruguaiana, RS, Brasil. Biodiversidade Pampeana, PUCRS, v.1, n.1, p. 35-45, 2003.

BARBIERI, G. Dinâmica da reprodução de cascudo, Rineloricaria latirostris Boulenger (Siluriforme, Loricariidae) do Rio Passa Cinco, Ipeúna, São Paulo. Revista Brasileira de Zoologia, Curitiba, v. 11, n. 4, p. 600-615, 1994.

BAZZOLI, N.; MESQUITA, T. L.; SANTOS, G. B.; RIZZO, E. Análise comparativa da reprodução de Astyanax bimaculatus
(Pisces, Characidae) nos Reservatórios de Furnas, Marimbondo e Itumbiara. Bios, Lima, v. 6, p. 99-112, 1998.

CASTELlanOS, Z. Fauna de água dulce de la República Argentina. La Plata: Profadu, 1991. 146 p.

COLARES DE MELLO, J. S. Influência do fotoperíodo sobre a maturação ovariana de mandi Pimelodus maculatus Lacepede, 1803. Boletim Técnico do CEPTA, Pirassununga, v. 2, n. 1, p. 13-18, 1989.

COSTA, A. P. R.; ANDRADE, D. R.; VIDAL JUNIOR, M. V.; SOUZA, G. Biologia reprodutiva de fêmeas de piau-vermelho. Pesquisa Agropecuária Brasileira, Brasília, v. 40, n. 8, p. 789795, 2005.

DIAS, J. F.; PERES-RIO, E.; CHAVES, P. T. C.; ROSSIWONCTSCHOWSKI, C. L. D. B. Análise macroscópica dos ovários de teleósteos: problemas de classificação e recomendações de procedimento. Revista Brasileira de Biologia, São Carlos, v. 58, n. 1, p. 55-69, 1998.

HIRSCHMANN, A.; BERNHARDT FIALHO, C.; GRILLO, H. C. Z. Reprodução de Hemiancistrus punctulatus Cardoso \& Malabarba, 1999 (Siluriformes: Loricariidae) no sistema da laguna dos Patos: uma espécie de ambiente lótico frente às alterações provocadas por represamentos. Neotropical Biology and Conservation, São Leopoldo, v. 6, n. 3, p. 250-257, 2011.

LIMA-JUNIOR, S. E.; GOITEIN. R. Condition factor and gonadal cycle of females of Pimelodus maculatus (Osteichthyes, Pimelodidae) in Piracicaba river (SP, Brazil). Boletim do Instituto de Pesca, São Paulo, v. 32, n. 1, p. 87-94, 2006.

MARCUCCI, K. M. I.; ORSI, M. L.; SHIBATTA, O. A. Abundância e aspectos reprodutivos de Loricariichthys platymetopon (Siluriformes, Loricariidae) em quatro trechos da represa Capivara, médio rio Paranapanema. Iheringia, Série Zoologia, Porto Alegre, v. 95 , n. 2, p. 197-203, 2005.

MEGA, D. F.; BEMVENUTI, M. A. Guia didático sobre alguns peixes da Lagoa Mangueira, RS. Cadernos de Ecologia Aquática, Rio Grande, v. 1, n. 2, p. 1-15, 2006.

MONTOYA-BURGOS, J. I. Historical biogeography of the catfish genus Hypostomus (Siluriformes: Loricariidae), with implications on the diversification of Neotropical ichthyofauna. Molecular Ecology, Oxford, v. 2, n. 1, p. 1855-1867, 2003.

PESSANO, E.; AZEVEDO, C. TOMASSONI, D.; QUEROL, M. V.; QUEROL E. Dinâmica populacional, densidade e biomassa da ictiofauna da foz do arroio Felizardo, Bacia do rio Uruguai médio, Uruguaiana, RS. Biodiversidade Pampeana, PUCRS, v.2, n.1, p. 16-23, 2004.

QUEROL, M. V. M.; QUEROL, E. Reprodução de traíra Hoplias malabaricus (Pisces, Erythrynidae) na região de Uruguaiana, Rio Grande do Sul, Brasil. Hífen, Uruguaiana, v. 18, n. 34, p. 31-45, 1993.

QUEROL, M. V. M.; QUEROL, H.; GOMES, N. N. A. Fator de condição gonadal, índice hepatossomático e recrutamento como indicadores do período de reprodução de Loricariichthys platymetopon (Osteichthyes, Loricariidae), Bacia do rio Uruguai médio, Sul do Brasil. Iheringia, Série Zoologia, Porto Alegre, v. 92, n. 3, p. 79-84, 2002.

QUEROL, M. V. M.; QUEROL, E.; PESSANO, E. F. Influência de fatores abióticos sobre dinâmica da reprodução do cascudo viola Loricariichthys platymetopon (Isbrucker \& Nijssen, 1979) 
(Siluriformes, Locariidae) no reservatório da estância Nova Esperança, Uruguaiana, Bacia do Rio Uruguai, RS, Brasil. Biodiversidade Pampeana, Uruguaiana, v. 2, n. 1, p. 24-29, 2004.

RAMOS, L. A.; KONRAD, H. G. Biologia reprodutiva de Hemiancistrus sp. (Ostheichthyes, Loricariidae) do rio dos Sinos, RS. Boletim do Instituto de Pesca, São Paulo, v. 25, n. 1, p. 4550, 1999.

REIS, R. E.; KULLANDER, S. O.; FERRARI JR, C. J. Check list of the freshwater fishes of South and Central. América. Porto Alegre: EDIPUCRS, 2003. 742 p.

RIBEIRO, C. S.; MOREIRA, R. G. Fatores ambientais e reprodução dos peixes. Revista da Biologia, São Paulo, v. 8, p. 58-61, 2012.

VAZ DOS SANTOS, A. M.; ROSSI WONGTSCHOWSK, C. L. D. B.; FIGUEIREDO, J. L. Parâmetros da reprodução e relação comprimento-peso da merluza Merluccius hubbsi (Teleostei: Merlucciidae), estoque sudoeste brasileiro $\left(21^{\circ} \mathrm{S} 29^{\circ} \mathrm{S}\right)$, ano 2004. Boletim do Instituto de Pesca, São Paulo, v. 35, n. 1, p. 1-16, 2009.
VAZZOLER, A. E. A. M. Manual de métodos para estudos biológicos de população de peixes. Reprodução e crescimento. Brasília: CNPq Programa Nacional de Zoologia, 1981. 108 p.

VAZZOLER, A. E. A. M. Biologia da reprodução de peixes teleósteos: teoria e prática. Maringá: EDUEM/SBI/CNPq/Nupelia, 1996. 169 p.

VIANA, D.; WOLLF, L. L.; ZALESKI, T. Population structure and somatic indexes of Hypostomus cf. ancistroides (Siluriformes, Loricariidae) collected from the Bonito River, Ivaí river basin, Turvo, Paraná. Brazil Archive Biology and Technology, Curitiba, v. 51, n. 3, p. 493-502, 2008. 